\title{
APONECROSIS DE LA CORTEZA PIRIFORME EN RATAS ALBINAS SOMETIDAS A ADMINISTRACIÓN CRÓNICA DE BEBIDAS ALCOHÓLICAS ADULTERADAS
}

\author{
APONECROSIS OF THE PYRIFORM CORTEX IN RATS \\ SUBMITTED TO CHRONIC ADMINISTRATION OF \\ ADULTERATED ALCOHOLIC BEVERAGES
}

JOSÉ RAFAEL DÍAZ RUDAS

Universidad Norbert Wiener

\section{RESUMEN}

En nuestro país, el alcoholismo es un problema de salud pública por la alta morbi-mortalidad que produce. Las bebidas alcohólicas que se consumen son diversas, y existe preferencia por las bebidas alcohólicas adulteradas en las zonas urbanomarginales. Para esta investigación se utilizaron 60 ratas albinas, Rattus norvegicus, cepa Holtzman, de 8 semanas de edad y de sexo masculino; las que fueron distribuidas en 5 grupos de 12 y recibieron una dosis diaria de 5,06 gramos de etanol $/ \mathrm{kg}$ de peso corporal. Se emplearon las bebidas alcohólicas conocidas como "Tumbaloco" o pac: partes iguales de pisco, anisado y cognac (todos adulterados); "Penal" o pac $+c$ : partes iguales de pisco, anisado y cognac (todos adulterados) + cerveza; "Pisco": pisco autorizado; "Pisco + Cerveza": pisco autorizado + cerveza autorizada. Luego de 12 semanas, las ratas albinas fueron sacrificadas por el método de decapitación, se les extrajo el encéfalo y se fijó en formol al $10 \%$ tamponado a $4{ }^{\circ} \mathrm{C}$ por 7 días. Se realizó un corte coronal a nivel Bregma $+3 \mathrm{~mm}$ para estudiar la corteza piriforme utilizando la coloración Hematoxilina \& Eosina. En la corteza piriforme se encontró mayor densidad neuronal aponecrótica en los grupos experimentales, a predominio del grupo Pisco, en comparación al grupo blanco. Ello sugiere la presencia de algún compuesto químico presente en esta bebida y ausente en las otras bebidas alcohólicas adulteradas. Concluimos que la bebida alcohólica cuyo consumo crónico produce una mayor densidad neuronal aponecrótica en la corteza piriforme es el pisco de venta autorizada más barato del mercado. La ańadidura de cerveza en las bebidas alcohólicas adulteradas no aumenta ni disminuye la aponecrosis neuronal.

Palabras clave: bebida alcohólica adulterada, corteza piriforme, aponecrosis neuronal.

\section{ABSTRACT}

In our country, alcoholism is a public health problem because of the morbidity and mortality that it produces. Alcoholic beverages that are consumed are different, it exists a preference for adulterated alcoholic beverages in poor zones. 60 male Holtzman rats, Rattus norvegicus, 8 weeks of age were used. They were distributed into 
five groups of twelve rats each, which received a dose of 5.06 grams ethanol/kg body weight. We used the alcoholic beverages known as "Tumbaloco" or pac: equal parts of pisco, cognac, aniseed (all adulterated); "Penal" or $p a c+c$ : equal parts of pisco, cognac and aniseed (all adulterated) + beer; "Pisco": authorized pisco; and "Pisco + cerveza": authorized Pisco + authorized beer. After 12 weeks, the rats were sacrificed by the decapitation method, extracting the encephalon and fixing it in $10 \%$ buffered formalin at $4{ }^{\circ} \mathrm{C}$ for 7 days. A coronal cut was then performed at the Bregma +3 mm level to study the pyriform cortex using the H\&E stain. In the pyriform cortex we found a higher necrotic neuronal density in the experimental groups with a predominance of the Pisco group. This makes us think of some chemical compound present in this alcoholic beverage and absent in adulterated drinks. We conclude that the alcoholic beverage whose chronic consumption causes more neuronal aponecrosis in the pyriform cortex is the cheapest authorized pisco on the market. The addition of beer in adulterated alcoholic beverages does not increase or decrease neuronal aponecrosis.

Key words: adulterated alcohol beverages, pyriform cortex, neuronal aponecrosis.

\section{INTRODUCCIÓN}

El consumo de alcohol está vinculado a las costumbres de casi todas las civilizaciones. Su consumo en moderación es aprobado por todas las culturas, pero su abuso, reprobado.

El alcoholismo es un problema de salud pública por la morbi-mortalidad que produce; en nuestro país, las bebidas alcohólicas que se consumen son distintas en costa, sierra y selva, registrándose un mayor consumo de bebidas alcohólicas adulteradas en las zonas urbanomarginales de las grandes ciudades.

Se han realizado innumerables trabajos de investigación para comprender los efectos del etanol en el cerebro utilizando animales de experimentación; y la rata albina es el animal modelo a seguir. La corteza piriforme es considerada parte de la corteza prefrontal y se encuentra por debajo de la corteza orbitofrontal ¿Las ratas tienen corteza prefrontal? Según la teoría de Rose y Woolsey (1948), la corteza prefrontal es propia únicamente de mamíferos primates, la cual está ba- sada solo en criterios anatómicos. Según Kosmal et al. (1987), las características anatómicas prefrontal dorsolateral están presentes también en los perros; sin embargo, Preuss y Kaas (1999) afirmaron que la corteza prefrontal dorsolateral es una especialización netamente primate. Posteriormente Uylings et al. (2003), demostraron mediante estudios anatómicos y funcionales que en la rata albina no solo existe corteza prefrontal orbital y medial, sino también dorsolateral ${ }^{1-4}$.

El etanol, también llamado alcohol etílico, es un compuesto orgánico que presenta dos carbonos y el grupo funcional hidroxilo $\left(\mathrm{C}_{2} \mathrm{H}_{5} \mathrm{OH}\right)$, líquido incoloro de olor característico y sabor quemante que se obtiene por fermentación de la glucosa a través de un agente de fermentación (transformación de la glucosa en etanol). El etanol es absorbido rápidamente en el estómago e intestino delgado; el $10 \%$ es eliminado por los riñones, piel y pulmones. También existe la posibilidad de tener un metabolismo cerebral del etanol, ello queda 
sustentado por la existencia de diferentes sistemas enzimáticos capaces de metabolizarlo.

En el cerebro, el mapa enzimático es menos conocido que en el hígado, y parece ser un tanto diferente. Se ha descrito la presencia de citocromos pertenecientes al complejo enzimático Microsomal Ethanol Oxidizing System (MEOS), y, en concreto, se ha demostrado que son inducidos por el etanol, como ocurre en el hígado. Se sabe que la distribución cerebral de citocromos pertenecientes al complejo enzimático MEOS en humanos no es uniforme, concentrándose sobre todo en neuronas granulares y piramidales del córtex cerebral, células de Purkinje, giro dentado e hipocampo. Cantidades muy pequeñas de etanol son oxidadas por la aldehído deshidrogenasa en el cerebro hasta la generación local de acetaldehído, lo cual tiene importantes consecuencias funcionales. Esta producción de acetaldehído ha sido asociada con la aceleración de la lipoperoxidación y con los efectos tóxicos del etanol, y la alteración de las membranas neuronales 5 .

En el Cercado de Lima, específicamente en la zona denominada "El Hueco", existe una gran cantidad de establecimientos que venden al libre albedrío bebidas alcohólicas adulteradas a muy bajo costo y sin registro sanitario. Esto se debe a la pobreza extrema que rodea a un gran número de la población y a la ineficacia de las autoridades en la erradicación de la producción de dichas bebidas. Aquí podemos encontrar la venta de pisco, cognac y anisado, todos ellos adulterados. Los consumidores las mezclan y les dan peculiares nombres a sus bebidas: "Tumbaloco" (partes iguales de pisco adulterado + cognac adulterado + anisado adulterado), "Penal" (partes iguales de pisco adulterado
+ cognac adulterado + anisado adulterado + cerveza de venta autorizada ${ }^{6}$.

La muerte neuronal comúnmente ha sido delineada como necrosis, la cual se considera un proceso pasivo asociado con la pérdida de homeostasis iónica, fallo de integridad de membrana e hinchazón celular y de organelas ${ }^{7-11}$. Las neuronas rojas son la primera etapa de la cascada de muerte celular neuronal programada, pasando luego por la necrosis. La necrosis fue tradicionalmente pensada y definida como un suceso no programado, una forma accidental de muerte celular en respuesta a un estrés abrumador que es incompatible con la supervivencia celular. Sin embargo, evidencia reciente indica que la necrosis puede también ser regulada, y que células como las neuronas pueden morir por una forma de necrosis llamada necroptosis, aponecrosis o necrosis regulada. La aponecrosis es caracterizada morfológicamente por una hinchazón celular y eventual ruptura que vierte su contenido en el entorno, desencadenando así una inflamación. Las vías de señalización de aponecrosis se intersectan con la regulación de apoptosis ${ }^{12-16}$.

Muy poco se ha escrito sobre el consumo de bebidas alcohólicas adulteradas en nuestro país. Se piensa que los daños que ocasiona este tipo de bebidas alcohólicas se deben al alto contenido de etanol y/o metanol que pudieran estar presentes en su composición; sin embargo, no se han realizado estudios concluyentes al respecto, y se cuenta únicamente con trabajos realizados en otros países con realidades totalmente distintas a las nuestras, donde se encuentran alteraciones histológicas del sistema nervioso debidas al consumo de bebidas alcohólicas reglamentadas, las que tienen un contenido de alcoholes ya estandarizado. 
El objetivo del presente estudio es determinar cuál es el mecanismo de muerte neuronal y cuál es la bebida alcohólica adulterada que produce una mayor densidad neuronal necrótica de la corteza piriforme de ratas albinas, luego de su administración crónica.

\section{MATERIALES Y MÉTODOS}

\section{Materiales}

Muestra. Se utilizaron 60 ratas albinas, Rattus norvegicus, cepa Holtzman, de 8 semanas de edad, sexo masculino y de 100 a 140 gramos de peso corporal. El lugar de manutención y procedimientos fue el bioterio animal de la Escuela de Medicina Humana de la Universidad Norbert Wiener (UPNW); se alimentó a las ratas con cebada ( $10 \mathrm{~g}$ de ceba$\mathrm{da} / 100 \mathrm{~g}$ de peso corporal) y agua $a d l i-$ bitum. Cada rata albina fue colocada en una jaula funcional de malla metálica de $30 \times 30 \times 20 \mathrm{~cm}^{3}$. Permanecieron en el bioterio con 12 horas de luz/oscuridad y con una temperatura de $24+/-2{ }^{\circ} \mathrm{C}$.

Bebidas alcohólicas adulteradas. Se compraron 12 botellas de pisco adulterado, 12 botellas de cognac adulterado y 12 botellas de anisado adulterado. Fueron obtenidas en la ciudad de Lima, en las inmediaciones de "El Hueco", a un costo de 22 soles por 3 botellas de cada una de estas bebidas alcohólicas adulteradas (Figura 1).

Bebidas alcohólicas autorizadas. Se compraron 12 botellas de pisco de venta autorizada más barato del mercado y 12 botellas de cerveza Pilsen ${ }^{\circ}$.
Sonda orogástrica. Para la administración diaria de las bebidas alcohólicas se construyó una sonda de $12 \mathrm{~cm}$ a partir de una aguja de punción lumbar n. ${ }^{\circ} 20$, a la cual se le dio una curvatura; se le retiró el bisel y en su lugar se colocó una esfera de aleación de plata y bronce.

\section{Métodos}

Preparado a partir de las bebidas alcohólicas. Con las bebidas adquiridas se hicieron los siguientes preparados:

- "Tumbaloco", al que llamaremos pac: partes iguales de pisco adulterado + anisado adulterado + cognac adulterado.

- "Penal", al que llamaremos pac+c: partes iguales de pisco adulterado + anisado adulterado + cognac adulterado + cerveza Pilsen ${ }^{\circ}$.

- "Pisco", al que llamaremos Pisco: pisco de venta autorizada más barato del mercado.

\section{FIGURA $1^{*}$}

Bebidas alcohólicas adulteradas: pisco, anisado y cognac

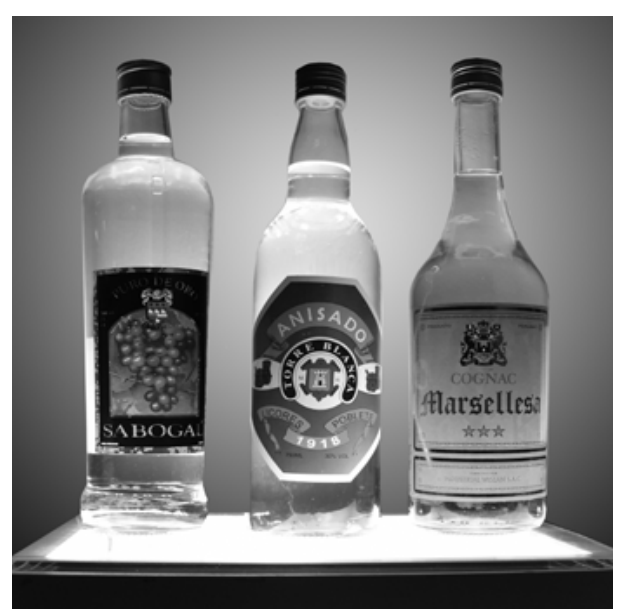

* Todas la figuras, tablas y anexos son producción propia. 
- "Pisco+cerveza", al que llamaremos Pis$c o+c: 3$ partes de pisco de venta autorizada más barato del mercado +1 parte de cerveza autorizada.

Aleatorización y distribución de la muestra. La muestra fue distribuida al azar en 5 grupos ( 1 grupo blanco y 4 grupos experimentales) de 12 ratas albinas cada una, a los cuales se les administró de manera diaria y durante 12 semanas: agua ad libitum (grupo blanco) y las bebidas alcohólicas anteriormente mencionadas (grupos pac, pactc, Pisco y Pisco+c).

Análisis de etanol en las bebidas alcohólicas adulteradas. Fue realizado en el Centro de información, control toxicológico y apoyo a la gestión ambiental (Сісотох) de la UNMSM, siguiendo el método de espectrofotometría UV-Vis. El contenido en grados de etanol en los preparados a partir de las bebidas alcohólicas es el siguiente:

- pac: $30,7^{\circ}$

- $\quad$ pac+c: $24,2^{\circ}$

- Pisco: $40^{\circ}$

- Pisco+c: $31,2^{\circ}$

Cálculo de la dosis. Para determinar el cálculo de la dosis, en esta investigación se ha tomado en cuenta el procedimiento y los resultados del estudio de Díaz ${ }^{5}$. Al grupo experimental se le administró una misma dosis de etanol, obtenida por extrapolación de una persona adulta de sexo masculino de $70 \mathrm{~kg}$ de peso corporal que consumió una botella diaria $(750 \mathrm{ml})$ de bebida alcohólica adulterada al $15 \%$ de etanol durante 7 ańos, cuyo resultado fue una dosis de 5,06 gramos de etanol $/ \mathrm{kg}$ de peso corporal.
$\mathrm{gOH} / \mathrm{kg}_{\text {murino }}=\mathrm{Vol} . \mathrm{X}^{\circ}$. $\rho .1 / 70 \mathrm{~kg} .4$

Vol : $750 \mathrm{~mL} \mathrm{SOL}$

$\mathrm{X}^{\circ} \quad: 15^{\circ}=15 \mathrm{~mL} \mathrm{OH} / 100 \mathrm{~mL} \mathrm{SOL}$

$\rho \quad: 0,7876 \mathrm{~g} \mathrm{OH} / \mathrm{mL} \mathrm{OH}$

$70 \mathrm{~kg}$ : Peso corporal humano

4 : Factor a multiplicar, según metabolismo basal, para obtener $\mathrm{kg}$ murino

La dosis letal $50 \%$ (LD50) en ratas albinas es de 7,06 g de etanol $/ \mathrm{kg}$ de peso corporal; nuestra dosis diaria fue inferior a esta. El volumen a administrar fue de 4 +/- $2 \mathrm{ml}$; el volumen máximo del estómago de una rata albina adulta es de $7 \mathrm{ml}$; y nuestro volumen también inferior a este.

$$
\mathrm{gOH} / \mathrm{kg}_{\text {murino }}=5,06
$$

Luego:

$$
\operatorname{Vol~mL}{ }_{A} \cdot X_{A}^{\circ}=\operatorname{Vol~mL}_{B} \cdot X_{B}^{\circ}
$$

$\mathrm{Vol} \mathrm{mL}_{\text {murino }}=4 . \mathrm{Vol} \mathrm{mL}_{\text {humano }} \cdot$ Peso $\mathrm{kg}_{\text {murino }} /$ Peso $\mathrm{kg}_{\text {humano }}$

Una de las mayores confusiones al administrar una dosis adecuada de etanol en ratas albinas es la ligereza con las que se revisan las condiciones experimentales al efectuar comparaciones entre diferentes trabajos experimentales. Las extrapolaciones que se intentan establecer entre los resultados de experimentos con ratas albinas a menudo son absurdas y en otras ocasiones muy difíciles de sustentar. El metabolismo basal, es decir, la producción de calor durante 24 horas en condiciones de reposo, al ser calculada por kilogramo de peso corporal resultó mayor 
mientras menor fuera el peso corporal del animal a estudiar, motivo por el cual la dosis se calculó por $\mathrm{m}^{2}$ de superficie corporal en lugar de kg de peso corporal. En conclusión, al comparar los resultados de los experimentos en el hombre y la rata albina, es más objetivo correlacionarlos con la energía liberada en función de la superficie corporal y no del peso corporal. En consecuencia, el metabolismo basal para la rata albina es aproximadamente 4 veces mayor que en el hombre, por lo que se le administró un volumen multiplicado por el factor 4 en comparación al ser humano (véase Anexo) ${ }^{17}$.

\section{Manipulación de la rata albina y} sondeo. Se sujetó suavemente a la rata albina colocando la palma sobre el dorso del animal de experimentación, con el dedo índice y medio alrededor del cuello, y los demás dedos alrededor del tórax. Una vez inmovilizada, se procedió a la introducción de la sonda orogástrica por el hocico hasta llegar a la luz del estómago. Posteriormente se administró el volumen de bebida alcohólica asignado (Figura 2).

Sacrificio del animal de investigación. El sacrificio fue por el método de decapitación. Previamente, la rata albina fue anestesiada con la bebida alcohólica a la cual fue sometida de manera crónica durante el estudio. El grupo blanco fue anestesiado con pisco de venta autorizada más barato del mercado.

Extracción del encéfalo. Se realizó en un tiempo menor a 4 minutos. Se inició con un corte sagital de la piel del cráneo con hoja de bisturí n. ${ }^{\circ} 22$, luego se cortó $2 \mathrm{~mm}$ de hueso occipital con tijera recta y posterior ruptura de calota craneana con pinza Kelly recta hemostática. Con una sonda acanalada se retiró el encéfalo y las meninges; fueron seccionados los pares craneales con hoja de bisturí n. ${ }^{\circ} 11$ para luego colocar el encéfalo en formol al $10 \%$ tamponado a $4{ }^{\circ} \mathrm{C}$. Se dejó fijar el encéfalo durante 7 días.

\section{Cortes coronales de $\mathbf{2} \mathbf{~ m m}$ del en-} céfalo. Se fabricó un aparato para cortes coronales paralelos de $2 \mathrm{~mm}$ usando una plancha de vidrio, dos láminas portaobjetos y silicona. Para el corte se utilizó una cuchilla para micrótomo de la marca Leica (Figura 3).

\section{Identificación de corteza pirifor-} me. Se utilizó como referencia las imágenes del atlas de Paxinos y un estereomicroscopio. La corteza piriforme se encontró en un corte anteroposterior: Bregma $+3 \mathrm{~mm}$.

Deshidratación, aclaramiento e inclusión. Proceso que se llevó a cabo en el Departamento de Neuropatología del Instituto Nacional de Ciencias Neurológicas (INCN). Se deshidrató la muestra utilizando soluciones de alcohol en orden creciente. Para el aclaramiento se utilizó butoxietano. Posteriormente se incluyó la muestra en parafina para proceder a realizar cortes de $8 \mu \mathrm{m}$ de espesor. Se montaron los cortes en láminas y se empleó la tinción Hematoxilina \& Eosina (H\&E).

Micrografiado a 400X. Se utilizó un microscopio biológico Eclipse $\mathrm{Ci}-\mathrm{L} \mathrm{Ni}$ kon con una cámara adaptada al fototubo del mismo. Cuatro campos de corteza piriforme fueron estudiados al azar por cada corte coronal cerebral de rata albina ${ }^{18}$.

Morfometría y estereología. Para la morfometría se utilizó el software JMicrovision versión 1.2.7. Para la estereo- 


\section{FIGURA 2}

Manipulación y sondeo de la rata albina

- Coger suavemente del cuerpo.

- Colocar palma sobre dorso.

- Dedo índice y medio alrededor del cuello.

- Demás dedos alrededor del tórax.
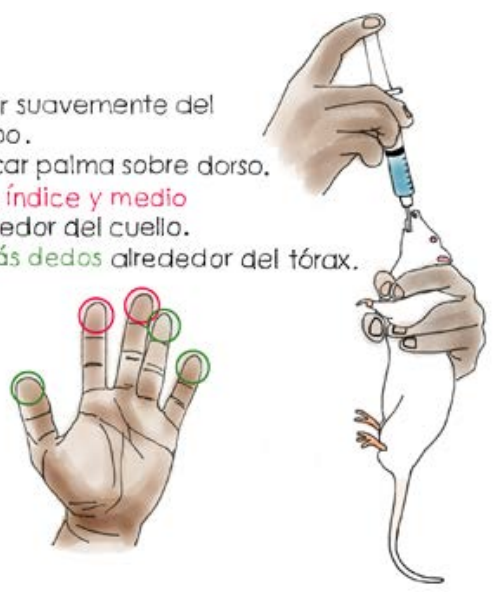

FIGURA 3

Corte coronal de encéfalo de rata albina

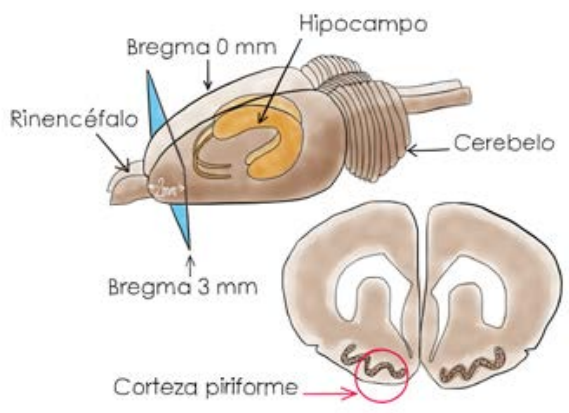

logía se utilizó el software ImageJ versión 1.51. Se realizó una evaluación de la densidad de área neuronal por campo (número de puntos que toca a una neurona / número de puntos totales del campo) por el método del point counting. El número de puntos totales que existen en un campo es de 70 (10 puntos en cada línea horizontal y 7 puntos en cada línea vertical), entre cada punto existe una distancia de $30 \mu \mathrm{m}$. La densidad de puntos es directamente proporcional a la densidad de área y, esta, a la densidad de volumen, de acuerdo con el principio de Delesse $\left(\mathrm{P}_{\mathrm{P}} / \mathrm{P}_{\mathrm{T}}=\mathrm{A}_{\mathrm{A}} / \mathrm{A}_{\mathrm{T}}=\mathrm{V}_{\mathrm{V}} / \mathrm{V}_{\mathrm{T}}\right)^{19,20}($ Figura 4$)$.

\section{FIGURA 4}

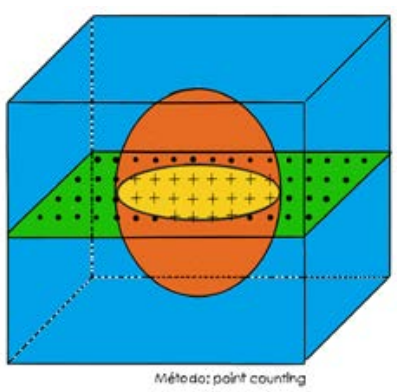

Principio de Delesse :

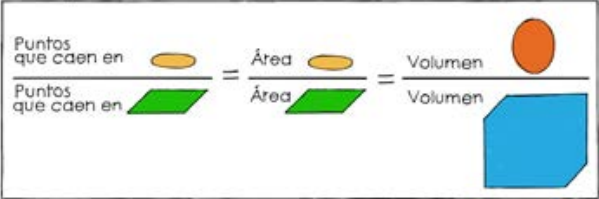

El principio de Delesse (1848) está basado en estructuras homogéneas. La razón entre puntos parciales y puntos totales es igual a la razón de longitudes, áreas y volúmenes.

La densidad neuronal necrótica corresponde al número de puntos que toca a una neurona necrótica dividido entre el número de puntos totales del campo. La densidad de área $\mathrm{A}_{\mathrm{A}}$ para neuronas necróticas fue estimada por la ratio $\mathrm{P}_{\text {necrótica }} / \mathrm{P}_{\text {Total }}$.

Análisis estadístico. Se utilizó el software IBM SPSS Statistics versión 22 y se realizó un análisis descriptivo utilizando el estadístico promedio. Se empleó el método de análisis de varianza (ANOVA) para comparar la media aritmética entre los 5 grupos (grupo blanco y grupos experimentales). Se utilizó el análisis de varianza pues los datos presentaron distribución normal. Para las comparaciones múltiples se utilizó la prueba de Scheffé. Se realizaron los promedios de cada grupo para determinar si habían diferencias significativas.

Ética. Se siguió las normas establecidas en la Ley 27265, que estipula las condiciones éticas para el manejo de animales de laboratorio. Se rechaza así cualquier acto de crueldad y se evita, de la 
misma manera, sufrimiento innecesario para el animal de laboratorio.

\section{RESULTADOS}

Estudio morfométrico y estereológico. Se realizó primero el estudio morfométrico para identificar la morfología de la muerte neuronal y de esa manera diferenciarlas de las neuronas normales.

Realizado el estudio morfométrico, se encontraron limitaciones a la nomenclatura de muerte neuronal, lo cual se hace evidente cuando los mecanismos de tipo apoptótico se asocian con morfología de tipo necrótico. De hecho, estos dos patrones de muerte celular coexisten en las neuronas del grupo experimental y del grupo blanco, identificándose formas morfológicas intermedias conocidas actualmente como aponecrosis. Encontramos muerte neuronal con hinchazón celular propia de la necrosis, como también muerte celular con disminución de volumen celular propio de la apoptosis. En adelante, en la presente investigación llamaremos aponecrosis a la muerte celular encontrada, no necrosis.

Se evidenció que no existe atrofia ni disminución de superficie de la corteza piriforme que enmascare la pérdida de neuronas. Al ser una estructura homogénea, se procedió a realizar el estudio estereológico para calcular la densidad de área neuronal aponecrótica.

En la corteza piriforme de ratas albinas encontramos la presencia de neuronas aponecróticas y normales tanto en el grupo blanco como en el experimental. Al microscopio de luz a 400x observamos que las neuronas aponecróticas presentan las siguientes carac- terísticas: color oscuro o intensa eosinofilia del citoplasma, pérdida de la citoarquitectura normal de los bordes neuronales, hinchazón y también condensación del cuerpo neuronal. Existe un aumento del número de neuronas aponecróticas en todos los grupos experimentales, a predominio de los grupos Pisco y Pisco+c (Figura 5).

\section{Densidad neuronal aponecrótica.} El análisis de varianza mostró diferencias altamente significativas $(\mathrm{p}<0,001)$. Con la prueba de Scheffé se observó que no hay diferencias significativas entre los grupos blanco, pac y pactc ni entre los grupos Pisco y Pisco+c; pero sí se obtuvieron diferencias significativas entre los grupos pac y blanco al compararlos con los grupos Pisco y Pisco+c. La más alta diferencia significativa se encontró entre el grupo blanco y el grupo Pisco. El mayor promedio de densidad neuronal aponecrótica se encontró en el grupo Pisco mientras que el menor promedio de densidad neuronal aponecrótica se encontró en el grupo blanco (Figuras 6 y 7 ).

En las bebidas alcohólicas a las que se les añadió cerveza, no se encontraron diferencias significativas al realizar el estudio de densidad neuronal aponecrótica entre los grupos pac y pactc, ni entre los grupos Pisco y Pisco+c (Tablas 1 y 2).

\section{DISCUSIÓN}

Hay creciente evidencia que implica la aponecrosis en la patogénesis de la enfermedad, incluyendo daño isquémico, neurodegeneración e infección viral, pero no se la ha relacionado aún con el alcoholismo crónico ni menos con el consumo crónico de bebidas alcohólicas adulteradas. 

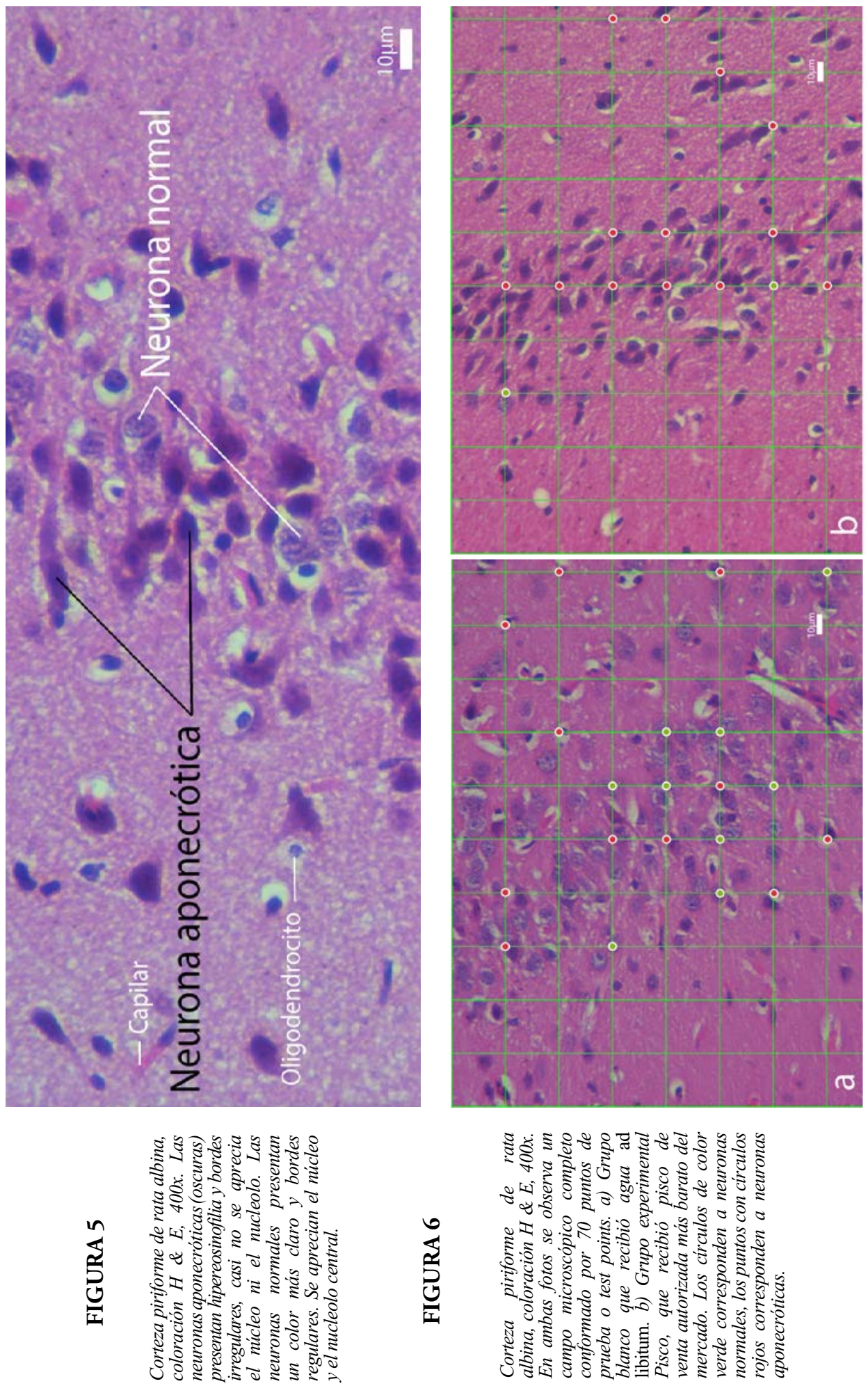

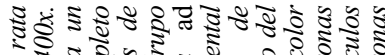

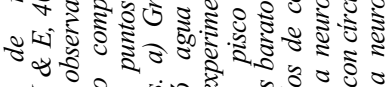

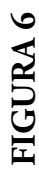

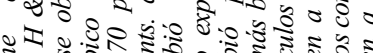

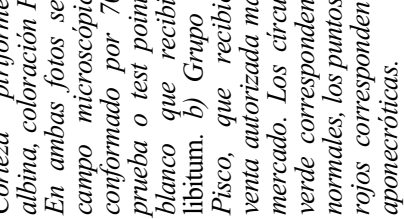




\section{FIGURA 7}

Corteza piriforme de rata albina, coloración Hematoxilina \& Eosina, 400x. Análisis estereológico de la densidad aponecrótica neuronal por el método del point counting y análisis morfométrico con abstracción de imagen en fondo negro; neuronas normales en color verde y neuronas aponecróticas en color rojo. a,c) Grupo blanco: disminución de la densidad aponecrótica neuronal. b,d) Grupo experimental Pisco*: aumento de la densidad aponecrótica neuronal.

* Pisco de venta autorizada más barato del mercado.
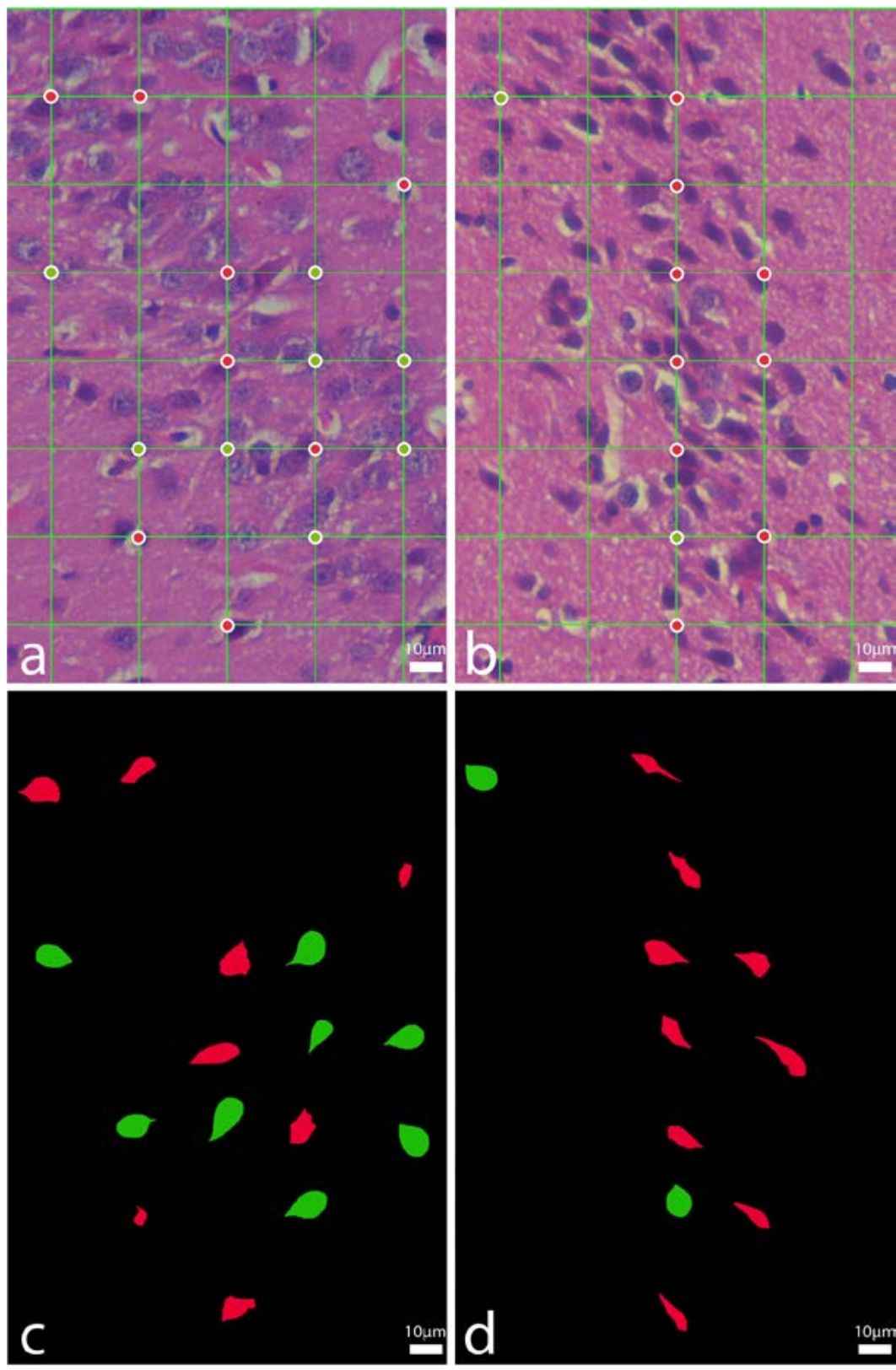
TABLA 1

Densidad neuronal aponecrótica

$\underline{\text { Scheffé } a, b}$

\begin{tabular}{lccc}
\hline & \multicolumn{2}{c}{ Densidad neuronal aponecrótica } \\
\cline { 3 - 4 } Bebidas alcohólicas & $\mathrm{N}$ & 1 & 2 \\
\hline Blanco & 12 & 8,92 & \\
Pac & 11 & 12,27 & 12,27 \\
Pac+c & 8 & 12,88 & 12,88 \\
Pisco+c & 12 & & 13,75 \\
Pisco & 12 & & 16,42 \\
\hline Significatividad & & 0,126 & 0,098
\end{tabular}

Se visualizan las medias para los grupos en los subconjuntos homogéneos.

a. Utiliza el tamaño muestral de la media armónica $=10,732$

b. Subconjunto para alfa $=0,05$

TABLA 2

Densidad neuronal aponecrótica

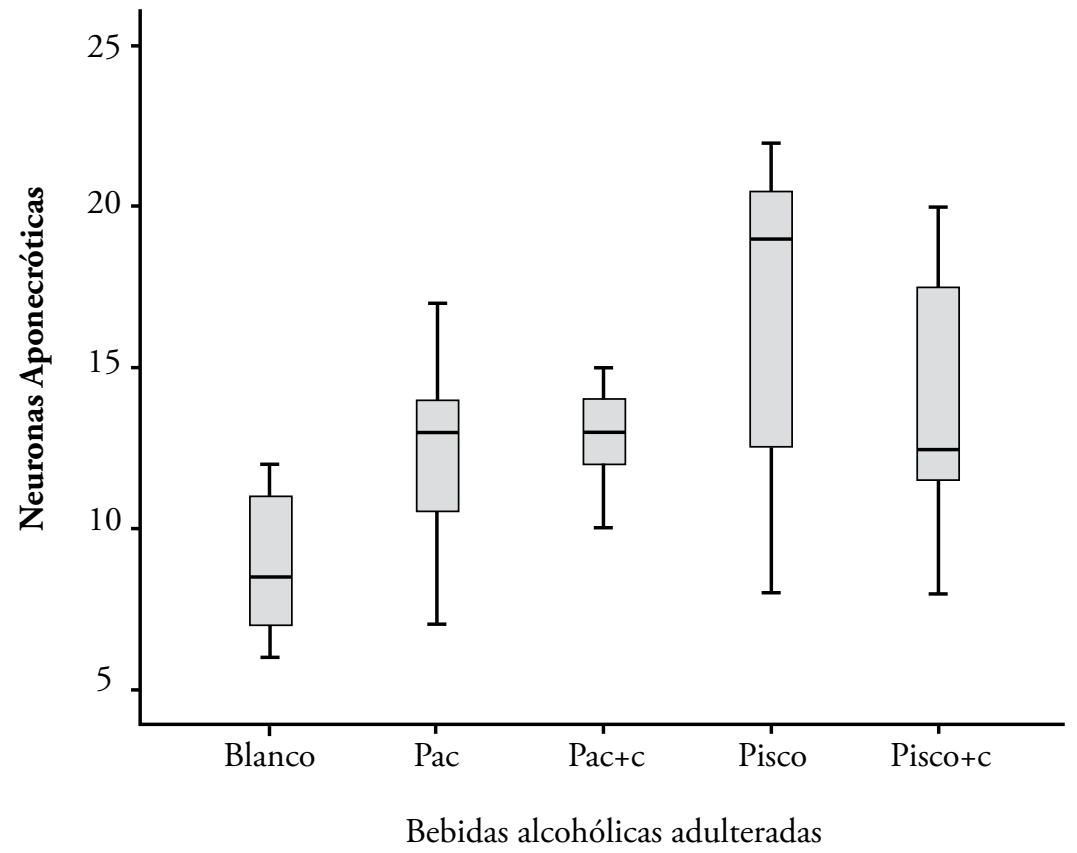


Se encontró mayor densidad neuronal aponecrótica en los grupos experimentales, grupos que fueron expuestos a una noxa: bebidas alcohólicas adulteradas, en relación al grupo blanco. En los cinco grupos, tanto en el blanco como en los experimentales, la aponecrosis como muerte celular está presente $\mathrm{e}^{21-27}$.

El marcado número de neuronas aponecróticas en los grupos experimentales puede explicarse por la cronicidad de la noxa, ya que todos los grupos experimentales recibieron las bebidas alcohólicas adulteradas durante doce semanas, de manera diaria, con una dosis única cercana al LD50 (dosis letal $50 \%$ ).

Recordar que no se administró ni la misma cantidad de volumen ni la misma cantidad de grados de etanol, en su lugar se administró la misma cantidad de gramos de etanol. La correlación año humano/días rata albina es de 1/11,8; por tanto, nuestro estudio es compatible con una persona adulta normal que consuma bebidas alcohólicas adulteradas de manera crónica por siete años ${ }^{28}$.

En un inicio se pensó utilizar un pisco de venta autorizada como control para hacer la comparación entre bebidas alcohólicas adulteradas y bebidas alcohólicas autorizadas. Utilizamos el pisco de venta autorizada más barato del mercado, y los análisis histológicos revelaron resultados que no habían sido considerados aun como posibilidad: el grupo con mayor densidad neuronal aponecrótica fue el grupo Pisco, lo cual nos hace pensar en algún compuesto químico presente en esta bebida alcohólica autorizada y ausente en las bebidas alcohólicas adulteradas.
¿Por qué el pisco de venta autorizada que hemos utilizado produce tanto daño neuronal? Tenemos que recordar que no hemos utilizado un pisco de calidad sino el pisco más barato del mercado; por ello, al encontrar alteraciones severas en la densidad neuronal, realizamos una búsqueda bibliográfica exhaustiva de documentos legales. Hallamos entonces que la marca del pisco usado fue cuestionada por problemas de calidad en 2014. Indecopi determinó que esta marca no cumplía con el reglamento de la denominación de origen, es decir que lo que se comercializaba no era pisco, bajo los estándares de calidad determinadas para ello, por lo que procedió a una denuncia de oficio por infracción de derechos de propiedad industrial, según resolución N. ${ }^{\circ}$ 3021-2014/CSD-INDECOPI. En consecuencia, se sancionó a esta marca con una multa equivalente a 10 UIT y se le prohibió usar indebidamente la denominación de origen para esa producción.

El análisis por medio de la cata del pisco de venta autorizada más barato del mercado por un experto demostró que este pisco fue adulterado en su variedad Quebranta, no la aromática. Así, este pisco no corresponde a su denominación de origen pues aparentemente pertenecia a la variedad aromática, por el olor despedido luego de unos minutos en los que se servía y dejaba volatilizar los alcoholes superiores. Poseía un olor característico a madera, que no debería estar presente, lo cual puede explicarse en la presencia de cantidades anormales de metanol. Además, al colocar unas gotas de este pisco bajo la lengua, producía ardor intenso en la mucosa oral, lo que podría además reafirmar la presencia de alcoholes dañinos. 
El pisco tiene tres partes en su destilación: la cabeza, el cuerpo y la cola. La cola es básicamente vapor de agua que no debería incluirse en la bebida. La cabeza posee alcoholes dañinos y es la parte que debería desecharse; al parecer, este pisco poseía algo de la cabeza, lo cual explicaría su precio y los daños neuronales encontrados.

El pisco de venta autorizada más barato del mercado ocasiona una mayor densidad neuronal aponecrótica por las consideracionaes siguientes:

a) la mala destilación que no eliminaría los otros alcoholes distintos al etanol (metanol y alcoholes superiores);

b) la presencia de altas cantidades de etanol $\left(40^{\circ}\right)$;

c) ser la única bebida alcohólica que no se mezcló con otras bebidas alcohólicas adulteradas; por tanto, no disminuyó su concentración de etanol ni su concentración de otros alcoholes distintos al etanol.

La bebida alcohólica adulterada pac presenta básicamente alcohol rectificado y otros compuestos químicos que no son alcoholes (colorantes y saborizantes), necesarios en el proceso de adulteración. El alcohol mal destilado produce más daño que el alcohol rectificado u oficinal (alcohol que contiene un $96 \%$ de etanol y no lleva indicador o marcador que le confiera mal sabor y olor), pues la rectificación del alcohol es posterior a la destilación del mismo y elimina en mayor cantidad todos los alcoholes diferentes al etanol ${ }^{29}$.
En Pisco + c y pactc la ańadidura de cerveza no tiene ningún efecto sobre la densidad neuronal aponecrótica, y esto puede ser explicado por la baja concentración de etanol en la cerveza $\left(5^{\circ}\right)$, por ser este un fermentado ${ }^{30}$ en comparación a los grupos Pisco y pac. Si la cerveza tuviera algún efecto, este quedaría enmascarado por los compuestos químicos nocivos distintos al etanol presentes en Pisco y en pac.

Para trabajos posteriores se sugiere analizar la cantidad de metanol en el pisco y, sobre todo, los demás alcoholes superiores presentes en dicha bebida alcohólica. Los resultados encontrados podrían deberse no solo a la presencia de etanol, sino de metanol u otros alcoholes.

Asimismo, se recomienda analizar la cantidad de metanol en la cerveza. Los alcoholes superiores son más nocivos que los compuestos químicos necesarios en la adulteración de las bebidas alcohólicas. No podemos afirmar que el daño encontrado en la corteza piriforme de ratas albinas será el mismo en todo el encéfalo, los daños son distintos dependiendo de la región a estudiar.

En conclusión, la muerte celular debido al consumo de bebidas alcohólicas es de tipo aponecrótica. La bebida alcohólica cuyo consumo crónico causa mayor densidad neuronal aponecrótica en la corteza piriforme es el pisco de venta autorizada más barato del mercado. Además, la añadidura de cerveza en las bebidas alcohólicas adulteradas no aumenta ni disminuye la densidad neuronal aponecrótica. 


\section{ANEXO}

\section{Tesis año 2015:}

Pac : $16,9^{\circ}$ etanol
Pac $+\mathrm{c}: 13,9^{\circ}$ etanol
Etanol $15 \%: 15,0^{\circ}$ etanol

Media de grados de etanol $=15^{\circ}$.

En 2015, Diaz estableció que una persona adulta de $70 \mathrm{~kg}$ de peso corporal con dependencia al alcohol consume una botella de $750 \mathrm{ml}$ con $15^{\circ}$ de etanol al dia. Histomorfometría de la capa granular orbitofrontal murina sometida a administración crónica de bebidas alcoholicas adulteradas (tesis de pregrado). UNMSM, Lima, Perú.

Cálculo de $\mathrm{gOH} / \mathrm{kg}_{\text {murino (gramos de etanol } / \mathrm{kg} \text { de peso corporal murino) }}$

\section{$\mathrm{gOH} / \mathrm{kg}_{\text {murino }}=$ volumen bebida. grados etanol. densidad etanol $.1 /$ peso corporal humano .4}

\section{$\mathrm{gOH} / \mathrm{kg}_{\text {murino }}=5,06$}

La dosis a administrar se encuentra por debajo del LD 50

\section{Investigación año 2017:}

Pac : $: 30,7^{\circ}$ etanol

$\mathrm{Pac}+\mathrm{c}: 24,2^{\circ}$ etanol

Pisco : $40,0^{\circ}$ etanol

Pisco + c: $31,2^{\circ}$ etanol
Se utilizó la misma dosis encontrada en la tesis del año 2015: $\mathrm{gOH} / \mathrm{kg}_{\text {murino }}=5,06$

Los datos de volumen $(750 \mathrm{ml})$ y grados de etanol $\left(15^{\circ}\right)$ fueron una constante a utilizar.

Cálculo de $\mathrm{m} \mathrm{l}_{\text {bebida }} / \mathrm{g}_{\text {murino }}$ a administrar:

Volumen $\mathrm{ml}_{\mathrm{B}} / \mathrm{g}_{\text {murino }}=$ volumen $_{\mathrm{A}}$. grados $_{\mathrm{A}} \cdot \frac{1}{\text { grados }_{\mathrm{B}}} \cdot \frac{1}{\text { Peso } \text { ggnumano }} \cdot \frac{\text { Peso } \mathrm{g}_{\text {murino }}}{1000} \cdot 4$

Volumen $\mathrm{ml} / \mathrm{g}_{\text {murino }}=750 \mathrm{ml} \cdot \frac{15 \mathrm{ml} \text { etanol }}{100 \mathrm{ml}} \cdot \frac{100 \mathrm{ml}_{\mathrm{B}}}{\mathrm{X} \mathrm{ml} \mathrm{etanol}} \cdot \frac{1}{70 \mathrm{~kg}_{\text {humano }}} \cdot \frac{\text { Peso } \mathrm{g}_{\text {murino }}}{1000} \cdot 4$

\begin{tabular}{|c|} 
(este dato serả una constante) \\
ml etanol \\
ml bebida \\
$\mathrm{ml}$ bebida $/ \mathrm{kg}$ humano \\
$\mathrm{ml}$
\end{tabular}




\section{REFERENCIAS BIBLIOGRÁFICAS}

1. Rose JE, Woolsey CN. The orbitofrontal cortex and its connections with the mediodorsal nucleus in rabbit, sheep and cat. Res Publ Assoc Nerv Ment Dis 1948; 27: 210-32.

2. Stepniewska I, Kosmal A. Distribution of mediodorsal thalamic nucleus afferents originating in the prefrontal association cortex of the dog. Acta Neurobiol Exp 1986; 46: 311-22.

3. Preuss TM, Kaas JH. Human brain evolution. In: Zigmond MJ, Bloom FE, Landis SC, Roberts JL, Squire LR, editors. Fundamental of neuroscience. San Diego, CA: Academic Press; 1999. p. 1283-311.

4. Uylings H, Groenewegen H, Kolb B. Do rats have a prefrontal cortex? Behav Brain Res 2003; 146: 3-17.

5. Díaz R. Histomorfometría de la capa granular orbitofrontal murina sometida a administración crónica de bebidas alcohólicas adulteradas. Lima: Universidad Nacional Mayor de San Marcos, 2015.

6. Dos Anjos M. The effects of alcohol in newborns. Einstein J Biol Med 2010; 8(3): 368-375.

7. Kumar et al. Robbins and Cotran's Pathological Basis of Disease. 9th ed. Philadelphia: Saunders/Elsevier; 2015.

8. Marti H. Erythropoietin and the hypoxic brain. J Exp Biol 2004; 207: 32333242.

9. Ziello J, Jovin I, Huang Yan. Hypoxia-Inducible Factor (HIF)-1 regulatory pathway and its potential for therapeutic intervention in malignancy and ischemia. Yale J Biol Med 2007; 80: 51-60.
10. Nitatori T et al. Delayed neuronal death in the CA1 pyramidal cell layer of the gerbil hippocampus following transient ischemia is apoptosis. J Neurosci 1995; 15(2): 1001-1011.

11. Grotta J et al. Stroke: pathophysiology, diagnosis, and management. 6th ed. Elsevier; 2016.

12. Zigmond M, Coyle J, Rowlqnd L. Neurobiology of Brain Disorders: Biological Basis of Neurological and psychiatric disorders. 1st ed. Academic Press; 2014.

13. Stoica B, Faden A. Cell death mechanisms and modulation in traumatic brain injury. Neurotherapeutics 2010; 7: 3-12.

14. Zhao $\mathrm{H}$ et al. Role of necroptosis in the pathogenesis of solid organ injury. Cell Death Dis 2015; 6(11): 3-10.

15. Longo D. Necroptosis. N England J Med 2014; 370(5): 455-465.

16. Goodlett C, Horn K. Necroptosis. Alcohol Res Health 2001; 25(3): 175-184.

17. Piño E, Gutiérrez Salinas J, MoralesGonzález J, Zentella M. ¿Es tóxico el alcohol? Mensaje Bioquímico 2003; 27: $121-140$.

18. Betton G. Photomicrographie, photographie au microscope. France: Photo-revue; 2012.

19. Delesse A. Procédé mécanique pour déterminer la composition des roches. Annals des mines 1848; 13: 379-388.

20. Weibel ER. Stereological methods. Practical methods for biological morphometry. Vol 1. London: Academic Press; 1979.

21. Becerra L, Pimienta H. Apoptosis neuronal: la diversidad de señales y de ti- 
pos celulares. Colomb Med 2009; 40: 124-33.

22. Nitatori T, et al. Delayed Neuronal Death in the CA1 Pyramidal Cell Layer of the Gerbil Hippocampus following Transient Ischemia Is Apoptosis. J Neurosci 2005; 15(2): 1001-1011.

23. Jimenez del Río M, Vélez-Pardo C. La apoptosis en las enfermedades neurodegenerativas: evidencias y controversias. Rev Neurol 2010; 32(9): 851-860.

24. Sanchez S, et al. Mecanismos de daño celular en enfermedades neurodegenerativas. Investigación en salud 2007; 9(3): 205-203.

25. Artal-Sanz M, Tavernarakis N. Proteolytic mechanisms in necrotic cell death and neurodegeneration. FEBS Letters 2005; 579: 3287-3296.
26. Kroemer G, et al. Classification of cell death: recommendations of the Nomenclature Committee on Cell Death 2009. Cell Death and Differentiation 2009; 16: 3-11.

27. Von Bernhardi R. Mecanismos de muerte celular en las enfermedades neurodegenerativas: ‘apoptosis o necrosis? Rev Chil Neuro-Psiquiat 2004; 42(4): 281-292.

28. Sengupta P. Laboratory rat: relating its age with human's. Int J Prev Med 2013; 4: 624-30.

29. O'neil MJ, editors. The Merck Index: An encyclopedia of chemicals and drugs. 15th ed. New Jersey: Merck; 2013.

30. Suárez M. Cerveza: Componentes y propiedades. Oviedo: Universidad de Oviedo, 2013.

\section{Agradecimientos}

El autor agradece a Palmer Hernández, Carolina Huayta, Geraldine Santos, María de los Ángeles Rivera (estudiantes de Medicina Humana de la Universidad Norbert Wiener), Alfredo Lora (docente de Medicina Humana de la Universidad Norbert Wiener), Nancy Rojas Instituto de Patología de la UNMSM, Frecia Astocóndor y Diana Rivas (servicio de Neuropatología del INCN), Harold Hernández (director del Centro de Investigación y Fondo Editorial de la Universidad Norbert Wiener) y Agustín Iza (Rector de la Universidad Norbert Wiener) por la ayuda prestada en la elaboración y redacción del presente artículo de investigación. 